\title{
The value of the Estermann zeta functions at $s=0$
}

\author{
by
}

\section{MAKOto IshiBashi (Kagoshima)}

1. Introduction. The Estermann zeta function $E_{u}(s, a / q)$ is defined by the Dirichlet series

$$
E_{u}\left(s, \frac{a}{q}\right)=\sum_{n=1}^{\infty} \sigma_{u}(n) e\left(\frac{a n}{q}\right) n^{-s}, \quad \operatorname{Re}(s)>\operatorname{Re}(u)+1,
$$

where $e(\alpha)=e^{2 \pi i \alpha}, a, q$ are integers with $q \geq 1,(a, q)=1$, and $\sigma_{u}(n)=$ $\sum_{d \mid n} d^{u}$. It is known that $E_{u}(s, a / q)$ can be continued analytically to the whole complex plane up to a double pole at $s=1$ ([1]). This function naturally occurs in the study of the exponential sums of the type

$$
D_{u}\left(x, \frac{a}{q}\right)=\sum_{n \leq x}^{\prime} \sigma_{u}(n) e\left(\frac{a n}{q}\right) \quad([4]-[6]),
$$

where $\sum^{\prime}$ means that if $x$ is an integer, then the term with $n=x$ in the sum is to be halved. We can easily get the explicit formula for these sums by applying Perron's formula, i.e.

$$
\begin{aligned}
D_{u}\left(x, \frac{a}{q}\right)= & \frac{1}{q}(\log x+2 \gamma-1-2 q \log x) x \\
& +E_{u}\left(0, \frac{a}{q}\right)+\frac{1}{2 \pi i} \int_{(-\varepsilon)} E_{u}\left(s, \frac{a}{q}\right) x^{s} s^{-1} d s
\end{aligned}
$$

where $\gamma$ is Euler's constant, and the integral is taken along the vertical line with $\operatorname{Re}(s)=-\varepsilon, \varepsilon>0$.

In this paper we shall evaluate the constants $E_{u}(0, a / q)$ in terms of the cotangent function in the case $u$ is an integer and determine the $\mathbb{Q}$-linear relations between $E_{u}(0, a / q)$, where $\mathbb{Q}$ denotes the rational number field.

1991 Mathematics Subject Classification: Primary: 11M41; Secondary 11J99. 
2. Constants $E_{j}(0, a / q)$. Let $B_{j}(x)$ be the $j$ th Bernoulli polynomial. The next lemma shows a relation between the values of $B_{j}(x)$ and the $j$ th derivative of $\cot \pi x$ at $x=a / q$.

Lemma ([2]). Let $q \geq 2,1 \leq a \leq q,(a, q)=1$. Then

$$
(j+1)\left(\frac{i}{2}\right)^{j+1} \cot ^{(j)}\left(\frac{\pi a}{q}\right)=q^{j} \sum_{k=1}^{q-1} e\left(-\frac{\pi a}{q}\right) B_{j+1}\left(\frac{k}{q}\right)
$$

for $j=0,1, \ldots$

Now this lemma implies

THEOREM 1. We have

$$
E_{j}\left(0, \frac{a}{q}\right)= \begin{cases}\frac{B_{j+1}}{2(j+1)}, & j \text { odd }, \\ \left(-\frac{i}{2}\right)^{j+1} \sum_{k=1}^{q-1} \frac{k}{q} \cot ^{(j)}\left(\frac{\pi a k}{q}\right)+\frac{1}{4} \delta_{j, 0}, & j \text { even },\end{cases}
$$

for $q \geq 2$, where $\delta_{j, 0}=1$ for $j=0$ and 0 otherwise. For $q=1$,

$$
E_{j}(0,1)=\frac{(-1)^{j+1} B_{j+1}}{2(j+1)} .
$$

Proof. For $\operatorname{Re}(s)>j+1$, substituting for $\sigma_{j}(n)$, we obtain

$$
\begin{aligned}
E_{j}\left(s, \frac{a}{q}\right) & =\sum_{n=1}^{\infty} \sigma_{j}(n) e\left(\frac{a n}{q}\right) n^{-s}=\sum_{m, n=1}^{\infty} e\left(\frac{a m n}{q}\right) m^{j-s} n^{-s} \\
& =\sum_{k, l=1}^{q} e\left(\frac{a k l}{q}\right) \sum_{m \equiv k(q)} \sum_{n \equiv l(q)} m^{j-s} n^{-s} \\
& =q^{j-2 s} \sum_{k, l=1}^{q} e\left(\frac{a k l}{q}\right) \zeta(s-j, k / q) \zeta(s, l / q),
\end{aligned}
$$

where in the final step we have used the Hurwitz zeta function $\zeta(s, x)=$ $\sum_{n=0}^{\infty}(n+x)^{-s}(0<x \leq 1)$. Then it follows from the analytic continuation of $\zeta(s, x)$ that

$$
\begin{aligned}
E_{j}(0, a / q)= & q^{j} \sum_{k, l=1}^{q} e\left(\frac{a k l}{q}\right) \zeta(-j, k / q) \zeta(0, l / q) \\
= & q^{j} \sum_{k, l=1}^{q-1} e\left(\frac{a k l}{q}\right) \zeta(-j, k / q) \zeta(0, l / q) \\
& +q^{j}\left(\sum_{k=1}^{q-1} \zeta(0,1) \zeta(-j, k / q)+\sum_{l=1}^{q} \zeta(-j, 1) \zeta(0, l / q)\right)
\end{aligned}
$$




$$
\begin{aligned}
= & \frac{q^{j}}{j+1} \sum_{l=1}^{q-1} B_{1}(l / q) \sum_{k=1}^{q-1} e\left(\frac{a k l}{q}\right) B_{j+1}(k / q) \\
& + \begin{cases}1 / 4, & j=0, \\
\frac{B_{j+1}}{2(j+1)}, & j \geq 1,\end{cases}
\end{aligned}
$$

after some computations using

$$
\zeta(-j, k / q)=-\frac{1}{j+1} B_{j+1}(k / q), \quad j \geq 0 .
$$

Changing the variable of summation $k$ to $q-k$ and using $B_{j+1}(1-x)=$ $(-1)^{j+1} B_{j+1}(x), B_{1}(l / q)=l / q-1 / 2$ and Lemma, we obtain our formula.

3. $\mathbb{Q}$-linear relations. In $[2], \mathrm{K}$. Girstmair gave a unified approach to the determination of all the $\mathbb{Q}$-linear relations between conjugate numbers in a cyclotomic field. Summarizing, his method is as follows: Let $\mathbb{Q}_{q}=\mathbb{Q}(\zeta)$ be the $q$ th cyclotomic field with $\zeta=e(1 / q)$ and let $G=\operatorname{Gal}\left(\mathbb{Q}_{q} / \mathbb{Q}\right)$ be its Galois group viewed as $(\mathbb{Z} / q \mathbb{Z})^{\times}$. We consider $\mathbb{Q}_{q}$ as a $\mathbb{Q} G$-module, where $\mathbb{Q} G$ denotes the group ring. For $b \in \mathbb{Q}_{q}$, the $\mathbb{Q}$-linear relations of the numbers $\sigma(b), \sigma \in G$, are determined by the annihilator $W_{q}(b)$ of $b$ in $\mathbb{Q} G$ defined by

$$
W_{q}(b)=\{\alpha \in \mathbb{Q} G: \alpha \circ b=0\},
$$

where $\alpha \circ b=\sum_{\sigma \in G} a_{\sigma} \sigma(b)$ for $\alpha=\sum_{\sigma \in G} a_{\sigma} \sigma \in \mathbb{Q} G$. It is known that any non-zero ideal $I$ in $\mathbb{Q} G$ is generated by the unique idempotent element $\varepsilon_{X}=\sum_{\chi \in X} \varepsilon_{\chi}$, written $I=\left\langle\varepsilon_{X}\right\rangle$, where

$$
\varepsilon_{\chi}=|G|^{-1} \sum_{\sigma \in G} \chi\left(\sigma^{-1}\right) \sigma, \quad X=\{\chi \in \widehat{G}: \chi(I) \neq 0\} .
$$

( $\widehat{G}$ denotes the character group of $G$.) He proves

Theorem A ([2]). The ideal $W_{q}(b)$ is generated by $\varepsilon_{X}$ with

$$
X=\{\chi \in \widehat{G}: y(\chi \mid b)=0\},
$$

where $y(\chi \mid b)$ are Leopoldt's character coordinates defined by

$$
y(\chi \mid b) \tau\left(\bar{\chi}_{f} \mid 1\right)=\sum_{\sigma \in G} \chi\left(\sigma^{-1}\right) \sigma(b)=\sum_{k=1}^{q} \bar{\chi}(k) \sigma_{k}(b),
$$

where $f$ means the conductor of $\chi, \chi_{f}$ is the primitive character modulo $f$ attached to $\chi$ and $\tau(\chi \mid k)$ is the $k$-th Gauss sum.

He also shows how to compute $\varepsilon_{X}$ explicitly for a special choice of $X$ and obtains, among over things, 
Theorem B ([2]). Let $j \geq 0$ and $q \geq 2$. Then

$$
W_{q}\left[i^{j+1} \cot ^{(j)}(\pi / q)\right]=\left\langle 1+(-1)^{j} \sigma_{-1}\right\rangle,
$$

with $X=\left\{\chi \in \widehat{G}: \chi\left(\sigma_{-1}\right)=(-1)^{j}\right\}$, where $\sigma_{k} \in G$ are such that $\sigma_{k}(\zeta)=\zeta^{k}$ for $(k, n)=1$, and $i=\sqrt{-1}$.

Similarly, in the case of the numbers $E_{j}(0, a / q), 1 \leq a \leq q$, with $(a, q)=$ 1 which are conjugate in $\mathbb{Q}_{q}$, we can prove

TheOREm 2. Let $q \geq 2$ be a prime power and let $j$ be an even integer. Then

$$
W_{q}\left[E_{j}(0,1 / q)\right]=\left\langle 1+\sigma_{-1}\right\rangle .
$$

P r o of. By virtue of Theorem A, we have only to compute the character coordinates of the numbers $E_{j}(0,1 / q)$. From the $\mathbb{Q} G$-linearity of $y(\chi \mid-)$ and the character coordinates for $i^{j+1} \cot ^{(j)}(\pi / q)$ ([2], Theorem 2), we have

$$
\begin{aligned}
y\left(\chi \mid E_{j}(0,1 / q)\right) & =\left(-\frac{1}{2}\right)^{j+1} \sum_{k=1}^{q-1} \frac{k}{q} \chi(k) y\left(\chi \mid i^{j+1} \cot ^{(j)}(\pi / q)\right) \\
& =\frac{-1}{j+1}\left(\frac{q}{f}\right)^{j+1} \prod_{p \mid q}\left(1-\frac{\bar{\chi}_{f}(p)}{p^{j+1}}\right) B_{1, \chi} B_{j+1, \chi_{f}},
\end{aligned}
$$

where $p$ runs through the prime factors of $q$.

Here $B_{n, \chi}$ denotes the generalized $n$th Bernoulli number, satisfying the relations

$$
B_{n, \chi}=m^{n-1} \sum_{a=1}^{m} \chi(a) B_{n}(a / m),
$$

where $m$ is the modulus of $\chi$. In the case of primitive character it is known [3] that for the principal character $\chi_{0}, B_{1, \chi_{0}} \neq 0$ and $B_{j+1, \chi_{0}}=0$, for even $j \geq 2$, and for non-principal $\chi$,

$$
\begin{cases}B_{j+1, \chi} \neq 0, & j \neq \equiv \delta_{\chi}(\bmod 2), \\ B_{j+1, \chi}=0, & j \equiv \delta_{\chi}(\bmod 2),\end{cases}
$$

where

$$
\delta_{\chi}= \begin{cases}0, & \chi \text { even } \\ 1, & \chi \text { odd }\end{cases}
$$

Further, we see that $B_{1, \chi} \neq 0$ for odd $\chi$ if the modulus of $\chi$ is a prime power. Hence, we get $X=\left\{\chi \in \widehat{G}: \chi\left(\sigma_{-1}\right)=1\right\}$, for $W_{q}\left[E_{j}(0,1 / q)\right]$, which is just the same as Theorem B. This completes the proof.

Theorem 2 implies 
TheOREM 3. For the numbers $E_{j}(0, a / q), 1 \leq a \leq q(a, q)=1$, q a prime power and $c_{a} \in \mathbb{Q}$ we have

$$
\sum_{(a, q)=1} c_{a} E_{j}(0, a / q)=0 \quad \text { if, and only if, } c_{a}=c_{q-a} \text { and } \sum c_{a}=0 .
$$

We see easily that

Corollary 1. The numbers $E_{j}(0, a / q), 1 \leq a \leq q / 2,(a, q)=1$, $q a$ prime power, $j$ even, are linearly independent over $\mathbb{Q}$.

Acknowledgements. The author wishes to thank the referee for his careful reading of the manuscript and his helpful suggestions.

\section{References}

[1] T. Estermann, On the representation of a number as the sum of two products, Proc. London Math. Soc. (2) 31 (1930), 123-133.

[2] K. Girstmair, Character coordinates and annihilators of cyclotomic numbers, Manuscripta Math. 59 (1987), 375-389.

[3] K. Iwasawa, Lectures on p-adic L-functions, Ann. of Math. Stud. 74, Princeton Univ. Press, Princeton, N.J., 1972.

[4] M. Jutila, On exponential sums involving the divisor function, J. Reine Angew. Math. 355 (1985), 173-190.

[5] I. Kiuchi, On an exponential sum involving the arithmetic function $\sigma_{a}(n)$, Math. J. Okayama Univ. 29 (1987), 93-205.

[6] Y. Motohashi, Riemann-Siegel Formula, Lecture Notes, University of Colorado, Boulder, 1987.

DEPARTMENT OF LIBERAL ARTS

KAGOSHIMA NATIONAL COLLEGE OF TECHNOLOGY

1460-1 SHINKO, HAYATO-CHO, AIRA-GUN

KAGOSHIMA 899-51, JAPAN

Received on 21.5.1994

and in revised form on 29.12.1994

$(2620)$ 